\title{
IDENTIFICATION OF THE PROCESS OF DYNAMIC STRETCHING OF THREADS IN WARP-KNITTING TECHNOLOGY
}

\author{
Aleksandra Prążyńska*, Zbigniew Mikołajczyk \\ Department of Knitting Technology, Lodz University of Technology \\ 116 Żeromskiego St., 90-924 Łódź, Poland \\ E-mail: aleksandra.prazynska@p.lodz.pl, zbigniew.mikolajczyk@p.lodz.pl
}

\begin{abstract}
:
The publication presents a theoretical study of the susceptibility of rheological models of threads to dynamic stretching in the context of modern, highly efficient textile technologies. Input parameters of the four analyzed models, the Kelvin-Voigt model, the Maxwell model, Standard model 2, and the Zener model, corresponded to the actual values of the coefficients of viscosity, elasticity, kinematic and dynamic loading, and stretching speed for the analyzed polyester silk threads produced in a knitting process, with knitting speeds from 700 to 1,600 courses/min. The research proves that the tested thread models behave differently in the stretching process-the Kelvin-Voigt model is sensitive to both the increases in kinematic loading and viscosity coefficient, Standard model 2 is only susceptible to dynamic stiffness, and the Zener model is significantly sensitive to kinematic loading. All responses of the models increase with the increase in stretching speeds. The obtained results indicate substantial "accuracy tolerance" in setting input parameters while identifying dynamics of the knitting process on warp-knitting machines.
\end{abstract}

\section{Keywords:}

rheological models, stretching threads, knitting on warp-knitting machines, elasticity and viscosity coefficient

\section{Introduction}

In numerous textile processes, such as spinning, winding, warping, weaving, knitting, and sewing, there are dynamic loadings affecting the threads caused by the mechanisms of the machines or non-uniform thickness of the threads themselves. The size and nature of tensions in the threads on knitting machines, regardless of their types, substantially affect the accuracy of the technological process as well as the properties of the produced knitted fabrics.

As a result of continuous efforts of the manufacturers of knitting machines, aiming at improving performance parameters of the devices by increasing the velocity of the loop-forming elements, the speed at which the tensions (loadings) in the threads increase also rises. Such loadings often lead to unexpected behavior of the threads during manufacturing processes.

Previous metrological studies concerning textile threads were based on static research that does not take into account the excitation speeds occurring in modern warp-knitting machines. This publication presents research results on the identification of the process of dynamic stretching of threads on warp-knitting machines in the aspect of the analysis of rheological models of the process, together with their empirical verification.

The novelty of the research on identifying the process of stretching textile threads in the technology of knitting on warp-knitting machines lies in determining the susceptibility of relevant rheological models of textiles. Sensitivity of the model of the described phenomenon of threads stretching dynamics was made dependent on the gradient of changes in real input parameters, including kinematic and dynamic elasticity modules, viscosity coefficient, and loading speed.

Owing to the significant research scope and comprehensive results, the publication has been divided into two parts:

- Part I: Theoretical analysis of the susceptibility of rheological models of the process of stretching textile threads.

- Part II: Experimental identification of the process of stretching threads, together with the verification of rheological models.

The subject of the first part of the publication is important from the point of view of gaining knowledge concerning quantitative impact of input data on the changes in threads response to dynamic loadings, in the modeling of the process of stretching textile threads (by rheological models). The aim of the research is to determine the influence of the alterations of the mechanical parameters of the threads on the sensitivity of changes in the output data.

The second part of the publication will attempt to answer the question of how to dynamically model the threads in the knitting processes, so as to be able to refer the theoretical calculations to the actual process conditions with maximum likelihood. 
Yarns are solid objects characterized by high complexity of mechanical properties. The behavior of yarns under the influence of externally applied forces is much more complex than the behavior of many other solids. Owing to the characteristic feature possessed by yarns time dependence of their properties, it will be most convenient to describe the discussed phenomena by using the principles of mechanics of viscoelastic bodies [1]. Only in case of very small forces affecting the yarns, elastic deformation can be observed.

A body loaded with a system of forces undergoes deformations, including both relative elongations and non-dilatational strains. The occurrence of mutual dependencies between the tension and deformation states was proven by experimental studies. Such dependencies are known as rheological equation of state [2]. Thus, in case of bodies whose length much exceeds their transverse dimensions, such as fibers or yarns, loaded in the longitudinal direction, this is a relationship between tension $\sigma$, elongation $\varepsilon$, and time $t$.

Rheological equation of state (RRS) for one-dimensional bodies is

$$
R(\sigma, \varepsilon, t)=0
$$

Most real objects possess all rheological properties to a certain extent. The bodies in which some properties have predominance over the others are called perfect bodies.

Rheological model that illustrates the formation, change, and disappearance of elastic deformations of the yarn is called Hooke's model. In this model, the tensions are proportional to the deformations. The examined properties indicate that Hooke's body is a perfectly elastic body.

The behavior of permanent deformations is illustrated by Newton's model. This model is a damper comprising a cylinder filled with liquid, inside which a piston with holes keeps moving. The examined properties indicate that Newtonian body is viscous, creeps with constant speed, the hysteresis loop is an ellipse, the energy is dispersed, and damping of body vibrations is very high.

Hook's and Newton's models, representing elastic and viscous properties, form the basis for building complex models, the so-called viscoelastic ones.

The Maxwell and Kelvin-Voigt two-part (two-parameter) models are mechanical models whose behavior under the influence of tensile force in a similar way describes the formation of viscoelastic deformations. They are formed by connecting basic Hook's and Newton's models in series and parallel. In the description of the creep phenomenon, it is most convenient to use the Kelvin-Voigt model. In the observation of the phenomenon of relaxation of internal tension, the Maxwell model is the most comfortable.

Single-parameter Hook's and Newton's models and twoparameter Kelvin-Voigt and Maxwell models demonstrate some rheological properties. Each of them describes one particular characteristic: Hooke's model describes elasticity, Newton's model describes viscosity, the Kelvin-Voigt model describes creep, and the Maxwell model describes relaxation.

More complex models of three, four, or more parameters are constructed in order to provide accurate description of real bodies, showing all the above-mentioned properties at the same time.

A parallel connection of the Maxwell model and Hooke's model constitutes a three-parameter model, called standard model of the first kind. It can be proven that such bodies are equivalent from the point of view of their properties, and their equation of state takes the following form:

$$
a \sigma+a_{1} \dot{\sigma}=b \varepsilon+b_{1} \dot{\varepsilon}
$$

The so-called standard model of the second kind, which is a parallel connection of the Maxwell and Newtonian body or a serial connection of the Kelvin-Voigt and Newton's models, is also a three-parameter model.

The analysis of the three-parameter model of the first kind, called the Zener model, makes it possible to gain more accurate knowledge of the phenomenon of forces relaxation and creep. The Zener model is a system of Maxwell's and Hooke's models connected in parallel.

A four-parameter model of the first kind is a serial connection of Maxwell's and Kelvin-Voigt's models, while the model of the second kind is a parallel connection of the two Maxwell models. Just like in case of the standard model, both fourparameter models are equivalent and their rheological equation of state takes the form:

$$
a \sigma+a_{1} \dot{\sigma}+a_{2} \ddot{\sigma}=b_{1} \dot{\varepsilon}+b_{2} \ddot{\varepsilon}
$$

The model possessing properties described by this equation is called Bürgers' model.

Over the course of time, some multiparameter models were formulated, which more accurately represent the behavior of fibers. An example can be Hoffmann's five-parameter rheological model. This model is a system of the standard model connected in series with the so-called Bingham element (parallel connection of the Newton's damper and the so-called mechanical slider), which illustrates the occurrence of the so-called coulombian friction $6 f$.

Another solution are integral models formed on the basis of well-known simple mechanical models, using the principle of superposition.

With regard to textile processes, the behavior of bodies described with the help of variability of deformations under the influence of tensile stress is defined with the help of various models.

In the model of a back-rest device, the author Kopias treats warp threads as viscoelastic bodies, describing them by the 
Kelvin-Voigt model [3].

According to Szosland and Czołczyński [4], the majority of textile raw materials can be classified into bodies in which the relaxation of tensions and deformations takes place under the influence of suddenly applied loading. These properties, according to the authors, can be presented by the so-called Zener standard linear model.

In the work of Sajdl [5], forces values in the threads were modeled using a three-parameter Zener model, while introducing the weft on the loom. The author determined the value of absolute viscosity on the basis of creep curves.

Kowalski adopted a rheological model featured by a standard Zener model to analyze dynamic forces in the threads on a weft-knitting machine [6]. Rheological parameters of the threads were identified on the basis of forces relaxation. Adopting the following conditions for the occurrence of such relaxation $\varepsilon=$ const., $\mathrm{d} \varepsilon / \mathrm{d} t=0$, he obtained an equation describing the process of forces relaxation:

$$
F=c \cdot \varepsilon+c_{1} \cdot \varepsilon \cdot e^{-\frac{k_{1}}{\eta}}
$$

In the article [7], Vangheluwe discussed the manner of the fabric edge displacement using the model for calculating elastic properties of warp yarns and fabrics. The author maintains that the differences in the relaxation of the warp yarn and the fabric will cause displacements of the fabric edge during relaxation. In modeling, the author uses a twostep procedure:

1. modeling of viscoelastic behavior of the warp yarn and the fabric

2. model of displacement of the fabric edge, selected from the models describing viscoelastic behavior.

Although the models of viscoelastic behavior of the yarns and fabrics provide good results, those concerning displacement of the fabric edge are unsatisfactory.

Deformations of the yarn observed during the application of tensile force are the resultant of constituent deformations. Bauer and Winkler [8] presented the components of deformation of the yarn, occurring as a result of application of a constant force and tension changes in the yarn subjected to constant elongation.

Reunan [9] conducted some tests of stretching polyamide monofilaments at high speeds for three ranges of stretching velocity from $\varepsilon \leq 102 \% / s$ to $\varepsilon \geq 104 \% / s$. The study was conducted for clamping length of $10 \mathrm{~mm}$. The above research made it possible to draw a conclusion that the main deformation course is similar for all stretching speeds; however, the obtained values of forces and elongations greatly depend on the speed of stretching.

In article [10], Zurek introduced a modified rheological model of a filament consisting of two parallel elements: I, a spring of linear stiffness $\mathrm{C} 1$; II, a spring of linear stiffness C2 connected in series with (a) a friction element of constant friction resistance $T$ and additional resistance $\mathrm{K} \mathrm{e}_{2}$ and (b) a piston of mass $m$ sliding in liquid of internal friction $H$.

Vangheluwe's model, composed of a Maxwell element of viscosity $\eta$ and a Hook's spring of elasticity coefficient $M$ connected in parallel with a nonlinear spring of elasticity coefficient $\mathrm{C}$, has been developed primarily for yarns. In order to obtain a model reflecting fiber properties, a new model was worked out by introducing an exponent in the exponential function, and a spring connected in parallel to the Maxwell element to reflect the nonlinear behavior identified (in the equation) by squared elongation.

Using the same criterion, which was used to transform the Vangheluwe model, Zurek's model was modified by taking into account the nonlinear behavior of the spring connected in series with the damper.

Mailler, Nemoz, and Hamelin [11] studied polyvinyl chloride reinforced with a polyester fabric with high tensile strength used in structures subjected to stretching or inflating (rafts, inflatable boats, inflatable rescue platforms). Simple tests of viscoelasticity were performed at different frequencies by means of DMTA (dynamic mechanical thermal analysis). In order to choose a good fitting model, Cole-Cole's diagrams were drawn based on experimental results and compared with the model ones. The obtained experimental results can be represented by a biparabolic Zener's model. This model can be constructed by connecting springs and dampers in series and/or parallel.

In the article [12], Lasič and Srdjak discussed the possibility of using Alfrey's model to analyze tensile deformation of double-warp-knitted fabrics. This model consists of three interconnected Kelvin's models. The results of experimental studies showed that Alfrey's model may be used for describing the behavior of double-warp-knitted fabrics during tension and relaxation stages. 


\section{Characteristics of the analyzed rheological models}

Table 1. Analyzed rheological models [13-15]

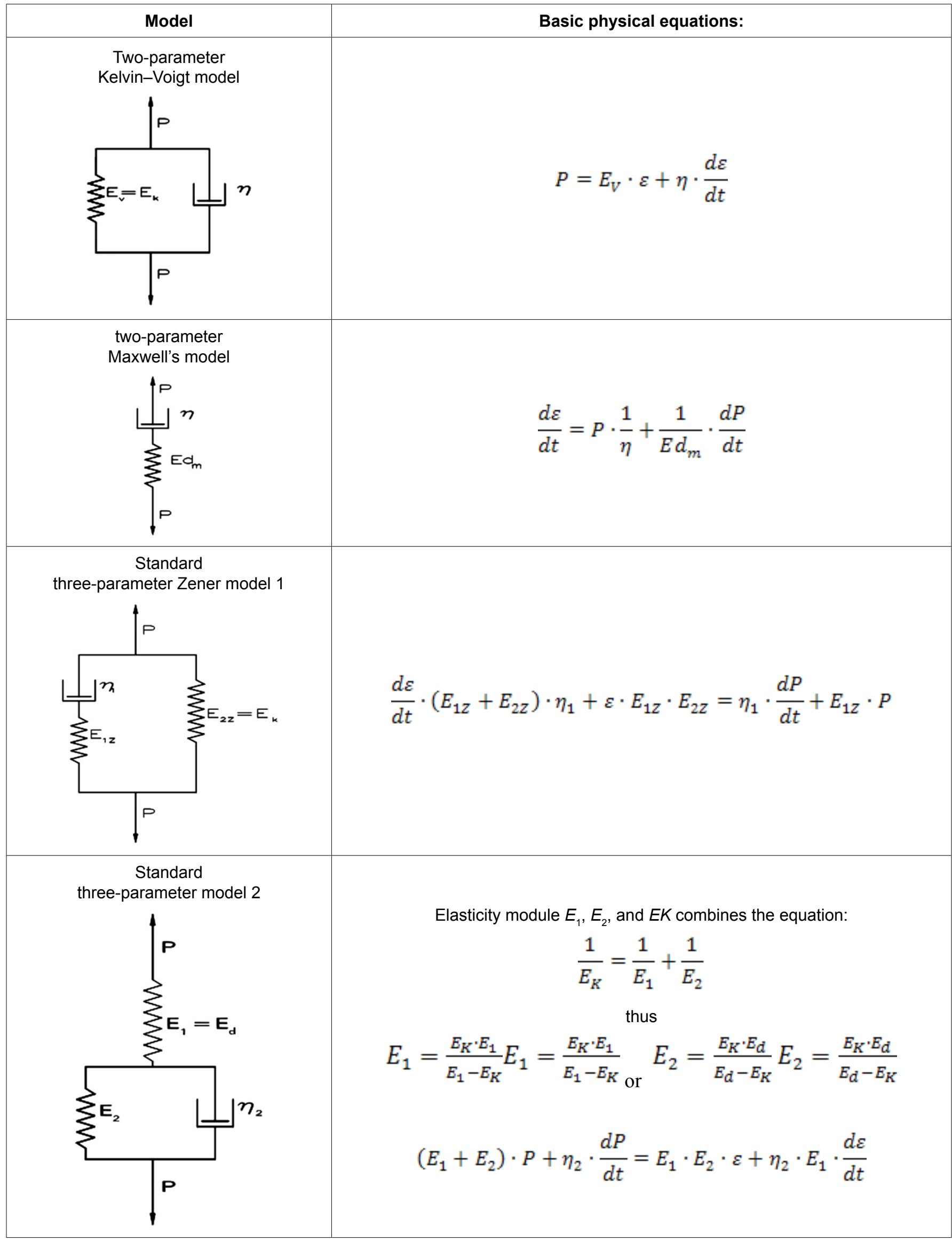




\section{Sensitivity of output data concerning forces in the thread in rheological models of the stretching processes to the input parameters}

The analysis was performed to determine the influence of the parameters of rheological models on the output values of time characteristics of forces in the threads.

The analysis was performed for the following ranges of input parameters:

- viscosity coefficient $\eta \in[20,420] \mathrm{cNs}$

- elasticity coefficient during kinematic loading $E_{\mathrm{K}} \in[1600$, 8400] cN,

- elasticity coefficient determined by dynamic loadings of the thread, calculated from the formula appropriate for the given rheological model:

Ed $\in[3170,16700] \mathrm{cN}$-the Maxwell model and Standard model 2,

Ed $\in[1100,10700] \mathrm{cN}$-the Zener model.

Dependencies $P=f(t)$ were performed for two values of the growth speed of relative elongation $\omega=0.51 / \mathrm{s}$ and $\omega=41 / \mathrm{s}$ and time $t \in[0,0.06]$ s corresponding to the time of stretching the threads for the range of the actual knitting speeds on warpknitting machines.

The analysis was performed in the environment of Mathcad.

\subsection{Kelvin-Voigt Model}

The solution of the equation of state for the discussed mode takes the form

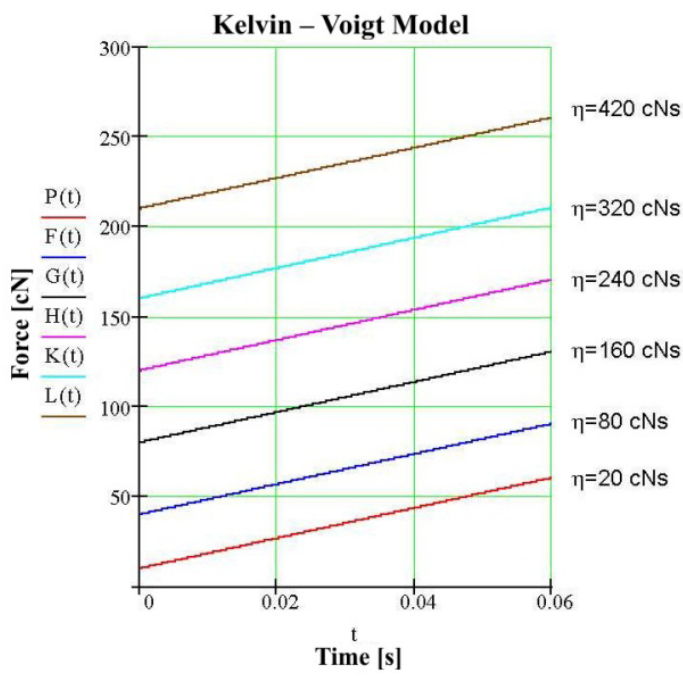

$\omega=0.51 / \mathrm{s}, E_{K}=1680 \mathrm{cN}$.

Figure 1. Characteristics $P=f(t)$ of the Kelvin-Voigt model for the viscosity coefficient $\eta \in[20,420] \mathrm{cNs}$.

$$
P(t)=E \cdot \omega \cdot t+\eta \cdot \omega
$$

For the first step of the analysis, the following input data were adopted: $E_{\mathrm{K}}=1680 \mathrm{cN}$,

$\omega=0.51 / \mathrm{s}$, and $\eta=20 \mathrm{cNs}$. In the following five steps, the basic viscosity value was multiplied to $\eta=420 \mathrm{cNs}$, which is the upper limit of the accepted values range for $h$. According to the dependencies $P(t) F(t) G(t), H(t), K(t)$, and $L(t)$ formulated in the program Mathcad, characteristics $P=f(t)$ of the KelvinVoigt model were performed for viscosity coefficient $\eta \in[20$, 420] cNs (Figure 1).

As in the above equation (5) $E^{\cdot} \omega=$ const and $\eta \cdot \omega=$ const, the resulting characteristics can be described by linear equation $y=k \cdot t+b$. For the initial value of viscosity coefficient $\eta$ $=20 \mathrm{cNs}$, the value of coefficient $b$ equals $10 \mathrm{cN}$ for $t=0$, which is illustrated by the first curve in the diagram (red). The subsequent curves are parallel. The difference in value between the subsequent characteristics determines the increase in coefficient $b$ in the equation. The inclination angle of the curves is determined by the coefficient $k=E \cdot \omega$. Identical nature of parallel lines is obtained for $\omega=41 / \mathrm{s}$. In case of increasing the value of relative elongation growth $w$, the value of maximum force for $\eta=420 \mathrm{cNs}$ also increases. For $\omega=0.5$ $1 / \mathrm{s}$, it equals $260.4 \mathrm{cN}$, and for $\omega=41 / \mathrm{s}$, it equals $2083.2 \mathrm{cN}$.

Subsequently, characteristics $P=f(t)$ of the Kelvin-Voigt model were analyzed for the elasticity coefficient during kinematic loading $E_{\mathrm{K}} \in[1680,8358] \mathrm{cN}$. As in the case of force dependence as a function of viscosity coefficient, the considered characteristics can be described by linear equation in the form $y=k \cdot t+b$ (Fig. 2). For the first step of the analysis, the following values were adopted: $E_{K}=1680 \mathrm{cN}, \omega=0.51 / \mathrm{s}$, and $\eta=20 \mathrm{cNs}$; in the remaining five steps, the basic value of

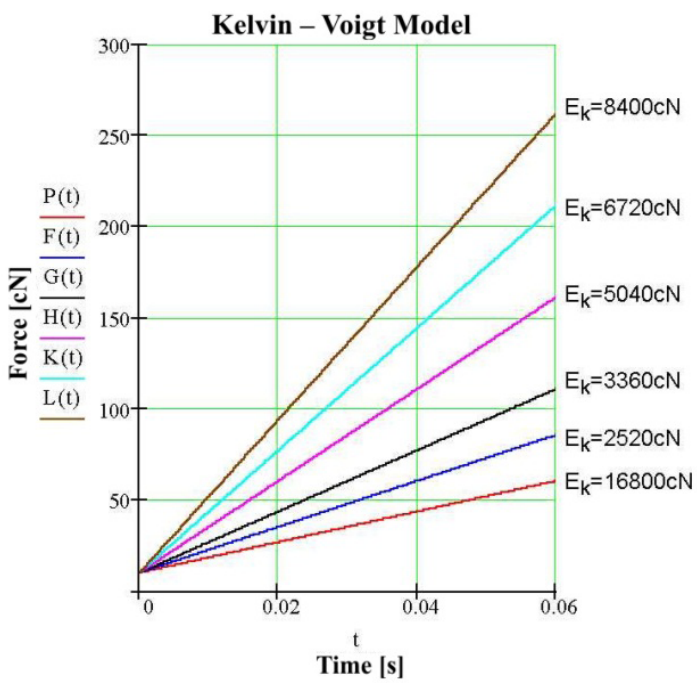

$\omega=0.51 / \mathrm{s}, \eta=20 \mathrm{cNs}$

Figure 2. Characteristics $P=f(t)$ of the Kelvin-Voigt model for the elasticity coefficient during the kinematic loading $E_{\mathrm{K}} \in[1680,8358]$ $\mathrm{cN}$. 
elasticity coefficient $E_{\mathrm{K}}$ was multiplied to $E_{\mathrm{K}}=8400 \mathrm{cN}$. With the increase in $E_{\mathrm{K}}$ coefficient, the coefficient $k$ in the equation also increases, as well as $\mathrm{Tg}$ of the inclination angle of the curves to the time axis. The course and variability of the characteristics for both accepted values of coefficient $w$ are the same. The graphs differ in maximum force value, for $\omega=0.51 / \mathrm{s}$, it equals $262 \mathrm{cN}$, and for $\omega=41 / \mathrm{s}$, it equals $2016 \mathrm{cN}$. The increase in force is greater for $E_{\mathrm{K}} \geq 5040 \mathrm{cN}$.

The nature of all curves is described by linear relationship. The growth dynamics of the force as a function of time is determined by elasticity coefficient $\mathbf{E}_{\mathbf{K}}$. The change in viscosity coefficient leads to the increase in the value by a fixed size of coefficient $b$, which in the case of presented graphs is shown by the arrangement of parallel lines. The model is sensitive to both the increase in stiffness modules $E_{K}$ and viscosity coefficients $\omega$. This sensitivity significantly increases with the increase in the forced stretching speed $\omega$.

\subsection{Maxwell model}

The solution of the equation of state for the Maxwell model takes the following form:

$$
P(t)=(F-\omega \cdot \eta) \cdot e^{\left(-t \cdot \frac{\xi}{\eta}\right)}+\omega \cdot \eta
$$

The first two diagrams (Figures 3 and 4 ) present characteristics $P=f(t)$ of the model for the viscosity coefficient $\eta=20-420$ cNs.

As in the case of the Kelvin-Voigt model, after adopting input data for the first step of the analysis, in the five subsequent steps, basic viscosity value was multiplied to $\eta=420 \mathrm{cNs}$.

As in equation (6), $F-\omega \cdot \eta=$ const., $-E / \eta=$ const., and

$\omega \cdot \eta=$ const., the obtained characteristics can be described by equation in the following form:

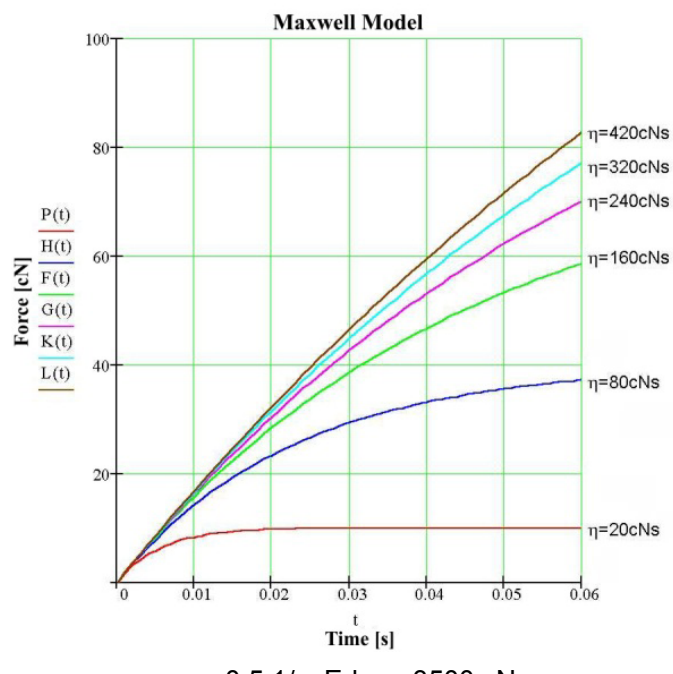

$\omega=0.51 / \mathrm{s}, \mathrm{Edm}=3500 \mathrm{cN}$

Figure 3. Characteristics $P=f(t)$ of the Maxwell model for the viscosity coefficient $\eta \in[20,420] \mathrm{cNs}$.

$$
y=a \cdot e^{b x}+c y=a \cdot e^{b x}+c
$$

where $\quad a=F-\omega \cdot \eta, b=-E / \eta, x=t, \quad c=\omega \cdot \eta$

Figure 3 illustrates the relationship of force as a function of viscosity coefficient for the following input data: $F=0$ and $\mathrm{Ed}_{\mathrm{m}}$ $=3500 \mathrm{cN}$.

Figure 4 shows the same characteristics for the increased value of coefficient $\mathrm{Ed}_{\mathrm{m}}=17000 \mathrm{cN}$, which is the upper limit of the accepted range for $E d_{m}$ values.

For the initial time values, the curves overlap. With the increase in $t$, the difference in value between the subsequent characteristics also increases, but for curves $\eta \in[160,420]$ $\mathrm{cN}$ in the period $t=0.02 \mathrm{~s}$, the divergence is insignificant. The sensitivity of the model increases with both, the increase in dynamic elasticity $\mathrm{Ed}_{\mathrm{m}}$ and viscosity $\eta$ for the times longer than $0.01 \mathrm{~s}$, and it substantially depends on the stretching speed $\omega$.

Figures 5 and 6 illustrate the relationship of force as a function of elasticity coefficient determined by dynamic thread loadings $\left(E d_{m} \in[3000,17000] c N\right)$. The first characteristic was performed for the following input data: $F=0$ and $\eta=20 \mathrm{cNs}$. The discussed system is not susceptible to changes in $\mathrm{Ed}_{\mathrm{m}}$ coefficient. While its value increases, the differences between the force values for the successive curves decrease, tending to zero. Figure 6 was made for the same input data as the previous one, except the value of the coefficient of viscosity which was changed to $420 \mathrm{cNs}$. Figure 6 shows force characteristics as a function of elasticity coefficient $E_{m}$ made for $\omega=41 / \mathrm{s}$. Significant changes in the impact of the $\mathrm{Ed}_{\mathrm{m}}$ coefficient are visible in the range $E d_{m} \in[3000,12000] c N$. Together with the increase in $\mathrm{Ed}_{\mathrm{m}}$ coefficient, the differences between force values for the subsequent curves decrease. For constant value of $\eta=420$ C cNs, sensitivity of the model increases, together with the increase in speed $\omega$.

The nature of all curves is described by an exponential function. The increase in force as a function of time is determined by

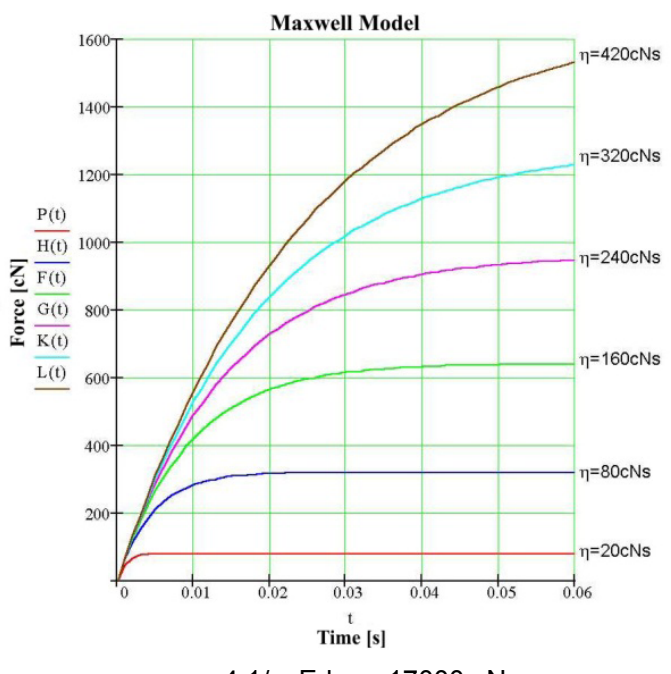

$\omega=41 / \mathrm{s}, \mathrm{Edm}=17000 \mathrm{cN}$

Figure 4. Characteristics $P=f(t)$ of the Maxwell model for the viscosity coefficient $\eta \in[20,420] \mathrm{cNs}$. 


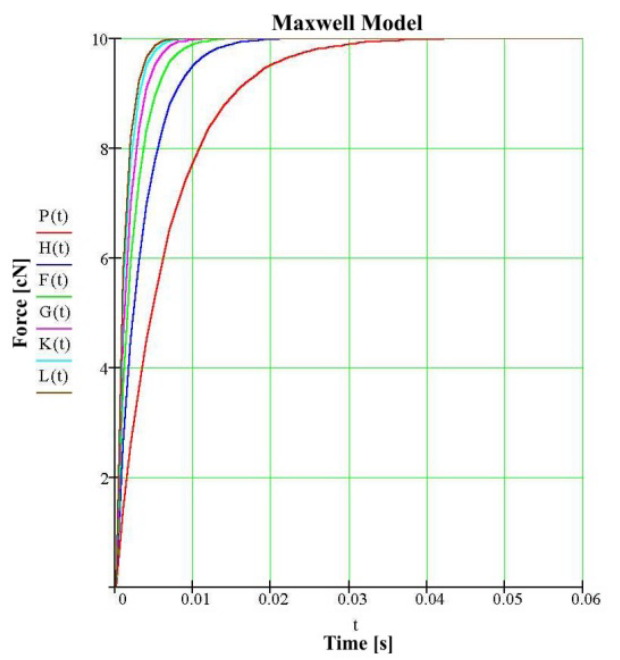

$\omega=0.51 / \mathrm{s}, \eta=20 \mathrm{cNs}$

$P(t)-\mathrm{Ed}_{\mathrm{m}}=3000 \mathrm{cN}, H(t)-\mathrm{Ed}_{\mathrm{m}}=6000 \mathrm{cN}, F(t)-\mathrm{Ed}_{\mathrm{m}}=9000 \mathrm{cN}$, $G(t)-{E d_{m}}_{m}=1200 \mathrm{cN}, K(t)-{E d_{m}}_{m}=15000 \mathrm{cN}, L(t)-{E d_{m}^{m}}_{m}=17000 \mathrm{cN}$.

Figure 5. Characteristics $P=f(t)$ of the Maxwell model for the elasticity coefficient determined by the dynamic loadings of threads $\mathrm{Ed}_{\mathrm{m}} \in[3000$, $17000] \mathrm{cN}$

coefficient $b$, that is, the ratio of Ed value to $\eta$. The system responds to the changes in the elasticity coefficient determined by dynamic loadings of threads and viscosity. An important susceptibility parameter is the stretching speed of the thread.

\subsection{Standard model 2}

The solution of the equation of state for the discussed model takes the following form:

$P(t)=P \cdot e^{\left(-a \cdot \frac{t}{a_{2}}\right)}+\omega \cdot \frac{\left(-b_{1} \cdot a_{2}+b_{1} \cdot a_{1} \cdot t+a_{1} \cdot b_{2}+e\left(-a_{1} \cdot \frac{t}{a_{2}}\right) \cdot b_{1} \cdot a_{2}-\theta\right.}{\left.\left(-a_{1} \cdot \frac{t}{a_{2}}\right) \cdot b_{2} \cdot a_{1}\right)}$

where $a_{1}=E_{1}+E_{2} \quad a_{2}=\eta \cdot \frac{a_{1}}{E_{1 z}}$

$$
b_{1}=E_{1} \cdot E_{2} \quad b_{2}=a_{2} \cdot E_{1}
$$

For the first step of the analysis, the following input data were adopted: $P=0, E_{1}=6765 \mathrm{cN}, E_{2}=5000 \mathrm{cN}, E_{1 \mathrm{Z}}=1000 \mathrm{cN}$, $E_{2 Z}=6000 \mathrm{cN}$, and $\eta=420 \mathrm{cNs}$. In the subsequent six steps, the basic value of viscosity coefficient was multiplied to $\eta=$ $420 \mathrm{cNs}$. An analysis of characteristics $P=f(t)$ of the Standard model 2

$\eta \in[20,420]$ cNs was carried out (Fig. 7).

In the time period $t \in[0,0.01] \mathrm{s}$, the curves overlap. Force $P$ increases with the increase in viscosity coefficient, but for curves $\eta \geq 160 \mathrm{cNs}$, the differences between the force values are minimal. For $\eta \geq 320 \mathrm{cNs}$, the curves take the form similar to a linear relationship. The behavior of the model is the same in both cases, the diagrams differ in maximum force value for $\eta=420 \mathrm{cNs}$.

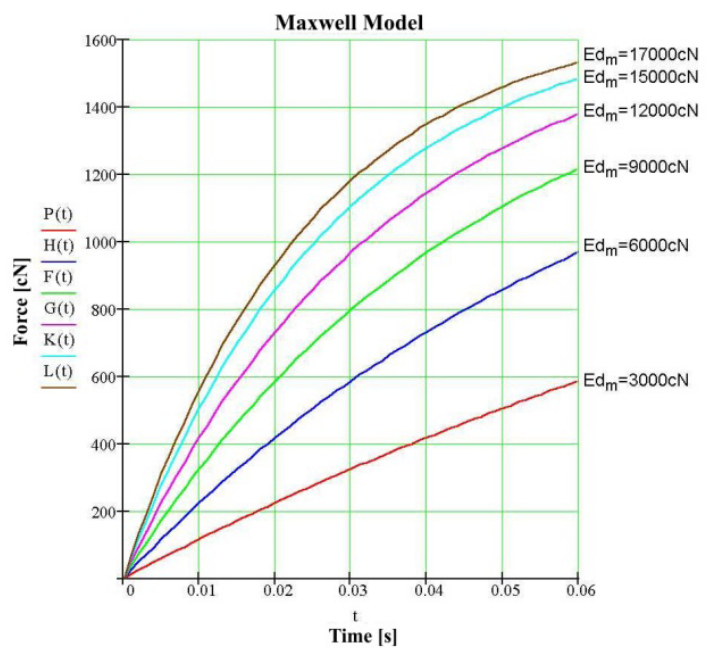

$\omega=41 / \mathrm{s}, \eta=420 \mathrm{cNs}$

Figure 6. Characteristics $P=f(t)$ of the Maxwell model for the elasticity coefficient determined by the dynamic loadings of threads $\mathrm{Ed}_{\mathrm{m}} \in[3000$, $17000] \mathrm{cN}$

Figure 8 is a relationship of force as a function of elasticity coefficient during kinematic loading $E_{\mathrm{K}} \in[1600,8400] \mathrm{cN}$. After adopting the following data for the first step: $P=0$,

$E_{1}=6765 \mathrm{cN}, E_{2}=1600 \mathrm{cN}, E_{1 \mathrm{z}}=1000 \mathrm{cN}$, and $\eta=200 \mathrm{cNs}$; in the successive six steps, the basic value $E_{\mathrm{K}}$ was multiplied. $E_{\mathrm{K}}$ coefficient has little effect on the characteristics of the Standard model 2. The curves take the form close to a linear relationship, and up to the time of $0.02 \mathrm{~s}$, they overlap.

Figure 9 is a relationship of force as a function of $E d_{s}$. Together with the increase in $\mathrm{Ed}_{\mathrm{S}}, \mathrm{Tg}$ of the inclination angle of the curves also increases, which can be described by a linear equation. The diagrams show a great influence of $\mathrm{Ed}_{\mathrm{s}}$ coefficient on the characteristics course.

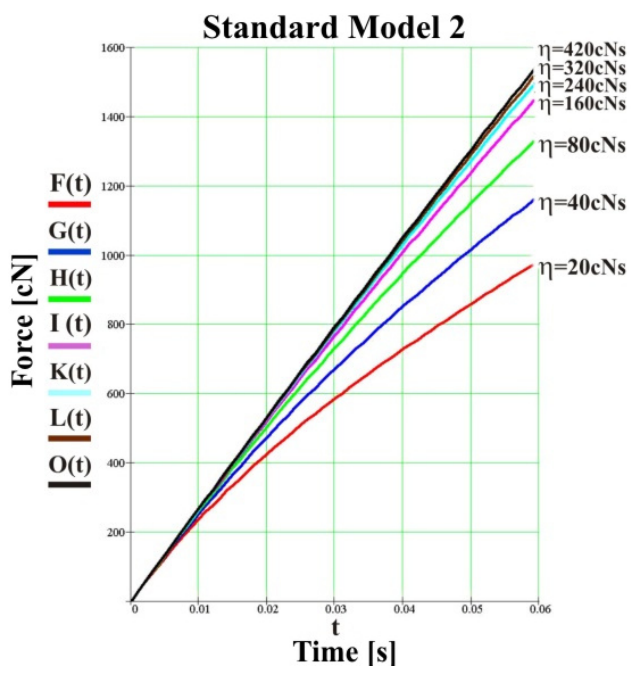

$\omega=41 / \mathrm{s}, E_{K}=5000 \mathrm{cN}, \mathrm{Ed}_{\mathrm{s}}=6765 \mathrm{cN}$

Figure 7. Characteristics $P=f(t)$ of the Standard model 2 for the viscosity coefficient $\eta \in[20,420] \mathrm{cNs}$. 


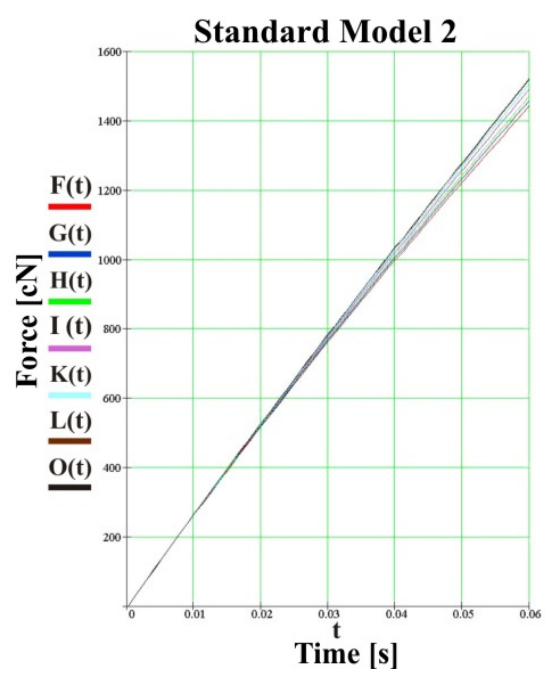

$\omega=41 / \mathrm{s}, \eta=200 \mathrm{cNs}, \mathrm{Ed}_{\mathrm{s}}=6765 \mathrm{cN}$

$F(t)-E_{K}=1600 \mathrm{cN}, G(t)-E_{K}=2400 \mathrm{cN}, H(t)-E_{K}=3200 \mathrm{cN}, I(t)-$ $E_{K}=4800$,

$K(t)-E_{K}=6400 \mathrm{cN}, L(t)-E_{K}=8000 \mathrm{cN}, O(t)-E_{K}=8400 \mathrm{cN}$.

Figure 8. Characteristics $P=f(t)$ of the Standard model 2 for the elasticity coefficient during the kinematic loading $E_{K} \in[1600,8400]$ $\mathrm{cN}$.

The function of dynamic tensions of the model has complex form, and the value of the time variable $t$ occurs in terms of linear dependence and exp. Significant susceptibility of the model is visible only for dynamic stiffness Ed, and slightly for viscosity coefficient $\eta$. The rule that sensitivity of input data increases with the increase of stretching velocity is maintained.

\subsection{Zener model}

The solution of the equation of state for the Zener model takes the following form:

$$
P(t)=P \cdot e^{-E_{1} \cdot \frac{t}{\eta}}+\eta \cdot \omega \cdot\left(1-e^{-E_{1} \cdot \frac{t}{\eta}}\right)+E_{2} \cdot \omega \cdot t
$$

For the first step of the analysis, the following input data were adopted: $P=0, E_{1}=6000 \mathrm{cN}, E_{2}=5000 \mathrm{cN}$, and $\eta=20 \mathrm{cNs}$. In the following five steps, the basic viscosity value was multiplied to $\eta=420 \mathrm{cN}$. On the basis of the obtained relationship, characteristics $P=f(t)$ of the Zener model were performed. The first analyses were performed for the increase in speed of relative elongation $\omega=0.51 / \mathrm{s}$, one for $\omega=41 / \mathrm{s}$. The nature and variability of the curves is the same in both cases; some differences are observed in the value of maximum force (Fig. 10). The differences in the values of force for the successive curves are very small. For time $t \geq 0.1 \mathrm{~s}$, they take the form of a linear relationship.

Figure 11 shows the relationship $P=f(t)$ for the elasticity coefficient during kinematic loading $E_{\mathrm{K}}$. For both the cases of stretching speed, the system is susceptible to changes in the coefficient in the range $E_{\mathrm{K}} \in[1600,8000] \mathrm{cN}$; above this value, the effect of $E_{\mathrm{K}}$ is negligible. For time $t \geq 0.1 \mathrm{~s}$, the curves can be described by linear equation. The behavior of the model for both $\omega=0.51 / \mathrm{s}$ and $\omega=41 / \mathrm{s}$ is similar. The graphs differ in the maximum force value.

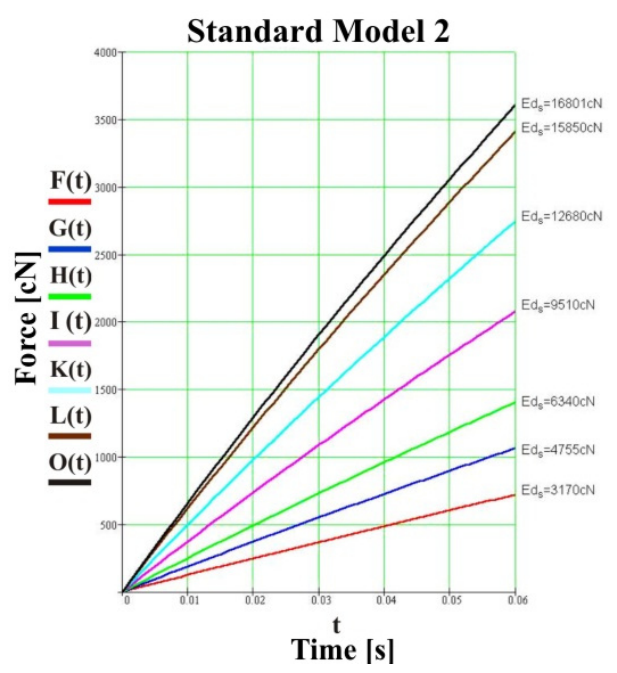

$\omega=41 / \mathrm{s}, \eta=200 \mathrm{cNs}, E_{K}=5000 \mathrm{cN}$

Figure 9. Characteristics $P=f(t)$ of the Standard model 2 for the elasticity coefficient determined by the dynamic loadings of threads $\mathrm{Ed}_{\mathrm{S}} \in[3170,16700] \mathrm{cN}$.

Figure 12 illustrates susceptibility of the Zener model by variability of the elasticity coefficient determined by dynamic loadings of threads $\mathrm{Ed}_{\mathrm{z}} \in[1100,10700] \mathrm{cN}$. The system is not susceptible to changes in $\mathrm{Ed}_{\mathrm{z}}$ value. In the time period $t \in[0,0.3] \mathrm{s}$, the difference between the force values are negligible. Above $t=0.3 \mathrm{~s}$, the characteristics overlap.

From the analysis performed for the adopted range of input data, it can be concluded that Zener model is sensitive to changes in the elasticity modulus during kinematic loading $E_{K}$ but does not react to changes in dynamic elasticity $E d$ and is only slightly influenced by changes in viscosity.

\section{Summary}

1. The behavior of ideal bodies subjected to stress is illustrated by Hook's mechanical models for perfectly elastic bodies and Newton's models for perfectly viscous ones. As most of the actual body possess to some extent all rheological properties, in order to describe them, it is necessary to build more complex models of two, three, four, or more parameters. Property analysis of these complex objects requires knowledge of their rheological equations of state, which may be difficult to solve because of the complexity of the models.

2. Rheological properties of textile materials (fibers, yarns, knitted, and woven fabrics) are most commonly modeled with the well-known mechanics, two- or three-parameter and integral systems, being a connection of Hook's body and Newton's damper. More complex models that incorporate nonlinear elastic bodies and friction elements with constant and nonlinear dry friction are also introduced. In modeling textile processes, including knitting on warp- and weft-knitting machines, weft insertion in weaving technologies, stretching synthetic filament threads, and phenomena destroying textile 


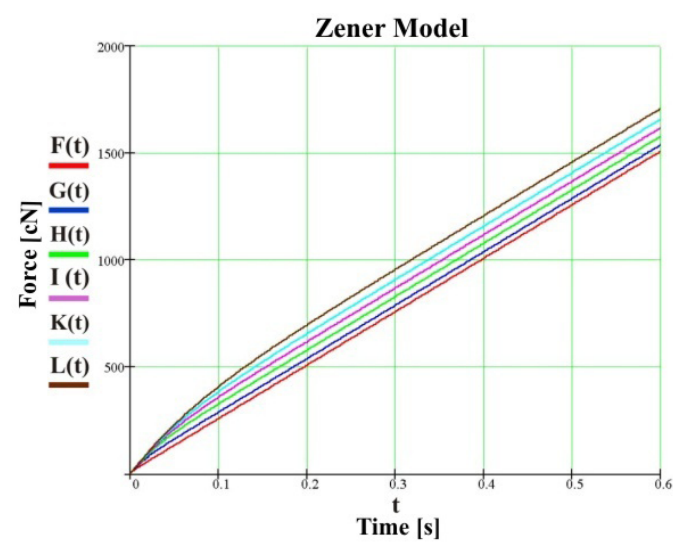

$\omega=0.51 / \mathrm{s}, E_{K}=5000 \mathrm{cN}, \mathrm{Edz}=6000 \mathrm{cN}$.

$F(t)-\eta=20 \mathrm{cNs}, G(t)-\eta=80 \mathrm{cNs}, H(t)-\eta=160 \mathrm{cNs}$, $I(t)-\eta=240 \mathrm{cNs}, K(t)-\eta=320 \mathrm{cNs}, L(t)-\eta=420 \mathrm{cNs}$.

Figure 10. Characteristics $P=f(t)$ of the Zener model for the viscosity coefficient $\eta \in[20,420] \mathrm{cNs}$.

materials, multielemental rheological models including the Kelvin-Voigt model, the Zener model in classical and parabolic approach, the nonlinear Maxwell model, or the Alfrey model turn out to be the most suitable to describe real phenomena.

3. As a result of theoretical research, susceptibility of selected models was determined:

- Kelvin-Voigt's model is sensitive to both the increase in stiffness modules $E_{\mathrm{K}}$ and viscosity coefficients $h$, wherein elasticity coefficient $E_{\mathrm{K}}$ defines the increase in dynamics of force over time, and change in viscosity coefficient leads only to the increase in force value by a fixed size of coefficient $b$.

- Maxwell's model shows that for initial values of viscosity coefficient, sensitivity of the output parameter is small, and for higher values, it increases rapidly. The system exhibits very high sensitivity to changes in the value of elasticity coefficient determined by dynamic loadings of the threads $\mathrm{Ed}_{\mathrm{m}}$,

- Standard Model 2 exhibits sensitivity only for dynamic stiffness $\mathrm{E}_{\mathrm{d}}$ and slightly for viscosity coefficient $h$, but does not react to any changes in elasticity module during kinematic loading $E_{\mathrm{K}}$,

- Zener's model is sensitive to changes in elasticity module during kinematic loading $E_{\mathrm{K}}$

and is slightly affected by changes in viscosity, but does not respond to any changes in dynamic elasticity Ed.

All the above sensitivities are enhanced with the increase in stretching speed $w$.

Low sensitivity of rheological models is a positive feature while describing physical phenomena. Too high sensitivity in that case is undesirable, as small changes in input parameters can cause major alterations of the evaluated characteristics of the output parameter. This makes it possible to define the accuracy (tolerance) of identifying empirical input parameters of the models.

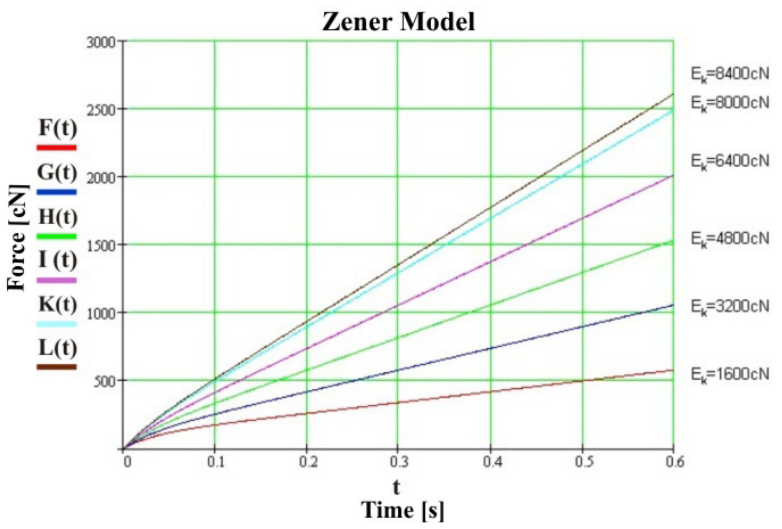

$\omega=0.51 / \mathrm{s}, \eta=200 \mathrm{cNs}, \mathrm{Edz}=6000 \mathrm{cN}$.

Figure 11. Characteristics $P=f(t)$ of the Zener model for the elasticity coefficient during the kinematic loading $E_{K} \in[1600,8400] \mathrm{cN}$.

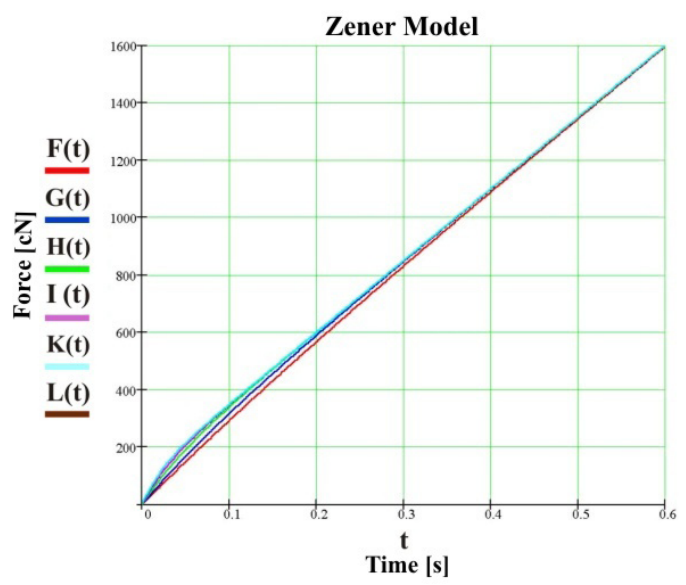

$\omega=0.51 / \mathrm{s}, \eta=200 \mathrm{cNs}, E_{\mathrm{K}}=5000 \mathrm{cN}$.

$F(t)-\mathrm{Edz}=1100 \mathrm{cN}, \mathrm{G}(t)-\mathrm{Edz}=2200 \mathrm{cN}, H(t)-\mathrm{Edz}=4400 \mathrm{cN}$, $I(t)-\mathrm{Edz}=6600 \mathrm{cN}, K(t)-\mathrm{Edz}=8800 \mathrm{cN}, L(t)-\mathrm{Edz}=10692 \mathrm{cN}$.

Figure 12. Characteristics $P=f(t)$ of the Zener model for the elasticity coefficient determined by the dynamic loadings of thread $\mathrm{Ed}_{\mathrm{z}} \in[1100,10700] \mathrm{cN}$.

\section{References}

[1] Urbańczyk G] Fiber Physics. Physical properties of fibers. (in Polish) Publishing House of Lodz University of Technology, Łódź 2002, p. 420.

[2] Kobza W., Gluza J., Technical mechanics and rheology. (in Polish) Handbook of TUL, Łódź 1991, p. 476.

[3] Kopias K. Technology of warp knitted fabrics. (in Polish) WNT, Warszawa 1984, p. 177.

[4] Szosland J., Czołczyński M. Propagation speed of the tension impulse in threads. (in Polish) Textile Review nr 27, Warszawa 1973, pp 122 - 127.

[5] Sajdl R. Modellierung der Beanspruchung beim Schusseintrag an Webmaschinen. Review of scientific and research works Moscow, Liberec, Łódź, Karl-Marx-Stat, 1985. 
[6] Kowalski K. Identification of dynamic forces in the threads on weft-knitting machines based on computer simulation and digital measurement technology. (in Polish) Science notebooks, Łódź 1991, pp. 17 - 22.

[7] Vangheluwe L. Displacement model of the fabric edge as a result of relaxation during stoppage time of the loom. International Conference IMTEX, Łódź, May 1995.

[8] Bauer, Winkler Relaxationsuntersuchungen an polymeren Festkorpern unter dem Aspekt der experimentellen Systemanalyse. Faserforschung und Textiltechnik 26, 1975 .

[9] Reuman R.D. Tests of polyamide monofilaments at high stretching speeds. Textile Review nr 9, Warszawa 1979 , pp. $494-498$.

[10] Żurek W. Modified rheological model of a filament. (in Polish) Scientific Conference of Textile Faculty Łódź 1998.
[11] P. Mailler, G. Nemoz*, P. Hamelin ${ }^{\star *}$ Long term behavior characterization of coated fabrics for architecture membrane under biaxial loading. Spring 1997, * Institut Textile de France D.R.LYON, ** L2M2S Université Claude Bernard Lyon I.

[12] Lasič V., Srdjak M. The Application of Alfrey's Model for the Stress - Deformation Analysis of Locknit Fabrics. July/ August 1995.

[13] Nowacki W. Theory of creep. (in Polish) Arkady Warsaw 1963, Issue I, 171p.

[14] Jakowluk A. Creep processes and fatigue in materials. (in Polish) WNT, Warsaw 1993, Issue I, ISBN 83-204-1542-X, 483p.

[15] Derski W., Ziemba S. Analysis of rheological models. (in Polish) Polish Scientific Publishers PWN, Warsaw 1968, Issue I, 